Pacific Journal of Mathematic 


\title{
ALGEBRAS SATISFYING THE DESCENDING CHAIN CONDITION FOR SUBALGEBRAS
}

\author{
Thomas P. Whaley
}

\begin{abstract}
In this paper we give a partial solution to the following problem of B. Jónsson:

(*) For which cardinals $m$ do there exist algebras of power $m$ having finitely many operations and satisfying the descending chain condition for subalgebras?
\end{abstract}

Of course a necessary condition for the existence of such an algebra is that there exist an algebra of power $m$ having finitely many operations and having no proper subalgebra of power $m$. The first such construction was by F. Galvin who constructed an algebra of power $\omega_{1}$ which satisfied the descending chain condition for subalgebras. It has been shown by Erdos and Hajnal [1] that for $n \in \omega$ there is an algebra of power $\omega_{n}$ which has finitely many operations and has no proper subalgebra of power $\omega_{n}$. Actually C. C. Chang [3] has shown that if an algebra exists of power $m$ having finitely many operations and having no proper subalgebra of power $m$, then such an algebra exists of power $m^{+}$. In $\S 2$ we modify this construction to show that if there is an algebra of power $m$ with finitely many operations and satisfying the descending chain condition, then there is such an algebra of power $\mathrm{m}^{+}$.

Erdos and Hajnal [1] also showed, under the assumption of the generalized continuum hypothesis, that for any cardinal $m$ there is a locally finite algebra of power $m^{+}$having finitely many operations and having no proper subalgebra of power $m^{+}$. In $\S 3$ we show that for $n \in \omega$ there is a locally finite algebra of power $\omega_{n}$ having finitely many operations and satisfying the descending chain condition for subalgebras.

2. General algebras. Before beginning the construction of the algebras we note the following relevant theorem of W. Hanf.

THEOREM 2.1. (Hanf [2], [4]). The lattice of subalgebras of an algebra with countably many operations is a compactly generated lattice in which each compact element contains at most countably many compact elements. Conversely, any such lattice can be realized as the lattice of subalgebras of a commutative loop in which each subalgebra is a subloop.

CoRollary 2.2. The following are equivalent:

(i) There exists a compactly generated lattice having $m$ compact elements in which each compact element contains at most countably 
many compact elements and which satisfies the descending chain condition (for elements).

(ii) There is an algebra of power $m$ having countably many operations and satisfying the descending chain condition for subalgebras.

(iii) There is an algebra of power $m$ having finitely many operations and satisfying the descending chain condition for subalgebras.

(iv) There is a commutative loop of power $m$ satisfying the descending chain condition for subalgebras.

THEOREM 2.3. If there is an algebra of power $m$ having finitely many operations and satisfying the descending chain condition for subalgebras, then there is an algebra of power $m^{+}$having finitely many operations and satisfying the descending chain condition for subalgebras.

Proof. Suppose we have such an algebra of power $m$. Using Corollary 2.2 we assume our algebra is of the form $A=\langle m ; f\rangle$ (identifying the cardinal $m$ with the set of all ordinals of cardinality less than $m$ ). Actually we could take $A$ to be a commutative loop, but these properties are not needed here. For each ordinal $\xi$ with $m \leqq$ $\xi<m^{+}$, let $\phi_{\xi}$ be a one-to-one map of $\xi$ onto $m$. We now define a binary operation $\bar{f}$ on $m^{+}$by

$$
\bar{f}\left(\eta_{0}, \eta_{1}\right)=\left\{\begin{array}{l}
f\left(\eta_{0}, \eta_{1}\right) \text { if } \eta_{0}, \eta_{1}<m, \\
\phi_{\eta_{0}}\left(\eta_{1}\right) \text { if } m \leqq \eta_{0} \text { and } \eta_{1}<\eta_{0}, \\
\phi_{\eta_{1}}{ }^{-1}\left(\eta_{0}\right) \text { if } \eta_{0}<m \leqq \eta_{1}, \\
0 \text { otherwise }
\end{array}\right.
$$

We show that $A^{\prime}=\left\langle m^{+} ; \bar{f}\right\rangle$ has the desired properties.

If $B$ is a subalgebra of $A^{\prime}\left(B \subseteq{ }_{s} A^{\prime}\right)$ then it is clear that $B \cap m$ is a subalgebra of $A$. Furthermore, if $m \leqq \xi \in B$ we can see that $m \cap B=\phi_{\xi}(\xi \cap B)$. To see this note that if $\eta \in \xi \cap B$ then $\phi_{\xi}(\eta)=$ $\bar{f}(\xi, \eta) \varepsilon m \cap B$ while if $\eta^{\prime} \in m \cap B$ then $\phi_{\xi}^{-1}\left(\eta^{\prime}\right)=\bar{f}\left(\eta^{\prime}, \xi\right) \in \xi \cap B$.

We now show that if $C \subset_{s} B \subset_{s} A^{\prime}$, one of the following three conditions must hold:

(i) $C \cap m \subset{ }_{s} B \cap m$,

(ii) $\Sigma C<\Sigma B$,

(iii) $\Sigma B \in B-C$.

Assume that $\Sigma C=\Sigma B$ and $\Sigma B \notin B-C$. Suppose first that $B$ has a largest member, $\beta$. Then $\beta=\Sigma B \notin B-C$ implying that $\beta \in C$. Thus $C \cap \beta \subset B \cap \beta$. We know that $C \cap m=\dot{\phi}_{\beta}(C \cap \beta) \subset \dot{\phi}_{\beta}(B \cap \beta)=B \cap m$. This leaves only the case where $B$ has no largest member. Take $\xi \in B-C$. If $\xi<m$, we have $C \cap m \subset B \cap m$. Therefore we assume 
that $m \leqq \xi<m^{+}$. Since $\Sigma B=\Sigma C>\xi$, there is a $\xi^{\prime} \in C$ with $\xi<\xi^{\prime}$. Then $\xi^{\prime} \cap C \subset \xi^{\prime} \cap B$ so $m \cap C=\phi_{\xi^{\prime}}\left(\xi^{\prime} \cap C\right) \subset \phi_{\xi^{\prime}}\left(\xi^{\prime} \cap B\right)=m \cap B$.

Suppose we have $A^{\prime} \supseteqq_{s} B_{0} \supseteqq_{s} B_{1} \supseteqq_{s} \cdots$. Clearly $\Sigma B_{0} \geqq \Sigma B_{1} \geqq \cdots$. There is some $k_{0} \in \omega$ so that $\Sigma B_{k_{0}}=\Sigma B_{k_{0}+1}=\cdots$. Also we know that

$$
A \supseteqq_{s} B_{k_{0}} \cap m \supseteqq_{s} B_{k_{0}+1} \cap m \supseteqq_{s} \cdots \text {. }
$$

Since $A$ satisfies the descending chain condition for subalgebras, there is a $k_{1} \geqq k_{0}$ so that $B_{k_{1}} \cap m=B_{k_{1}+1} \cap m=\cdots$. Assume now that $n_{1}<n_{2}<\cdots$ and that $B_{k_{1}} \supset B_{k_{1}+n_{1}} \supset B_{k_{1}+n_{2}} \supset \cdots$. Of the three conditions listed above, only (iii) applies to $B_{k_{1}+n_{2}} \subset_{s} B_{k_{1}+n_{1}} \subset_{s} A^{\prime}$. Thus $\Sigma B_{k_{0}} \in B_{k_{1}+n_{1}}-B_{k_{1}+n_{2}}$. Similarly, we get $\Sigma B_{k_{0}} \in B_{k_{1}+n_{2}}-B_{k_{1}+n_{3}}$. This contradiction completes the proof.

COROLlaRY 2.4. For $n \in \omega$ there is a commutative loop of power $\omega_{n}$ satisfying the descending chain condition for subalgebras.

3. Locally finite algebras. By a locally finite algebra we mean an algebra in which each finite subset generates a finite subalgebra. The following theorem characterizes the lattices of subalgebras of locally finite algebras in a manner somewhat analogous to Hanf's theorem.

THEOREM 3.1. The lattice of subalgebras of a locally finite algebra is a compactly generated lattice in which each compact element contains only finitely many compact elements. Conversely, any such lattice may be realized as the lattice of subalgebras of a locally finite algebra having one commutative binary operation.

Proof. Since the compact elements in the lattice of subalgebras of an algebra correspond to the finitely generated subalgebras and since each finitely generated subalgebra of a locally finite algebra is finite, it is clear that each compact element in the lattice of subalgebras of a locally finite algebra contains only finitely many compact elements.

Conversely, suppose $\langle L ;+, \cdot\rangle$ is a compactly generated lattice in which each compact element contains only finitely many compact elements. Let $L^{c}$ be the semilattice of compact elements of $L$. We know that $L$ is isomorphic to the lattice of ideals of $L^{c}$. We now define a commutative binary operation, $f$, on $L^{c}$ so that the subalgebras of $\left\langle L^{c} ; f\right\rangle$ are precisely the ideals of $\left\langle L^{c} ;+\right\rangle$ with the finitely generated subalgebras just the principal ideals. This will clearly complete the proof. For $a \in L^{c}$ let $\left\{a_{0}, a_{1}, \cdots, a_{n(a)}\right\}$ be the principal ideal of $\left\langle L^{c} ;+\right\rangle$ generated by $a$ with $a=a_{0}$ and $a_{i} \neq a_{j}$ if $i \neq j$. Define $f$ by 


$$
f(a, b)=\left\{\begin{array}{l}
a_{j+1} \text { if } b=a_{j} \text { with } j<n(a) \\
b_{j+1} \text { if } a=b_{j} \text { with } j<n(b) \\
a+b \text { otherwise. }
\end{array}\right.
$$

It is easy to check that the subalgebras of $\left\langle L^{c} ; f\right\rangle$ are as described above.

CoRollary 3.2. For any $m$ the following are equivalent:

(i) There is a compactly generated lattice having $m$ compact elements in which each compact element contains only finitely many compact elements and which satisfies the descending chain condition.

(ii) There is a locally finite algebra of power $m$ which satisfies the descending chain condition for subalgebras.

(iii) There is a locally finite algebra of power $m$ having one commutative binary operation and satisfying the descending chain condition for subalgebras.

TheOREM 3.3. For $n \in \omega$ there is a locally finite algebra of power $\omega_{n}$ which satisfies the descending chain condition for subalgebras.

Proof. The proof will be by induction on $n$. First we construct $A_{0}$ of power $\omega$. For each $m \in \omega$ define a unary operation $f_{m, 0}$ on $\omega$ by

$$
f_{m, 0}(n)=\left\{\begin{array}{l}
n-m \text { if } m \leqq n \\
0 \text { otherwise }
\end{array}\right.
$$

We then let $A_{0}=\left\langle\omega ; f_{m, 0}\right\rangle_{m \in \omega}$.

As an induction hypothesis we assume that we have

$$
A_{n}=\left\langle\omega_{n} ; f_{m, n}, \omega_{s}\right\rangle_{\substack{m \in \omega \\ s<n}}
$$

so that the following assertions are true of $A_{n}$ :

(1) $f_{m, n}$ is of rank $r(n)$ where $r(0)=1$ and $r(l+1)=2 r(l)+1$;

(2) $A_{n}$ is locally finite;

(3) For any $m \in \omega$ and for any $\eta_{0}, \eta_{1}, \cdots, \eta_{r(n)-1} \in \omega_{n}$, we have

$$
f_{m, n}\left(\eta_{0}, \cdots, \eta_{r(n)-1}\right) \leqq \cap\left(\left\{\eta_{i} \mid i \leqq r(n)-1\right\}-\left\{\omega_{s} \mid s<n\right\}\right)
$$

and $f_{0, n}\left(\eta_{0}, \eta_{0}, \cdots, \eta_{0}\right)=\eta_{0}$

(4) Given $\left\{\xi_{k} \mid k \in \omega\right\}$ a sequence of distinct members of $\omega_{n}$, there exist an $m \in \omega$ and $k_{0}, k_{1}, \cdots, k_{r(n)} \in \omega$ so that $k_{0}<\bigcap_{i=1}^{r(n)} k_{i}$ and

$$
f_{m, n}\left(\eta_{k_{1}}, \cdots, \eta_{k_{r(n)}}\right)=\xi_{k_{0}}
$$

where either $\eta_{k_{i}}=\xi_{k_{i}}$ or else $\eta_{k_{i}} \in\left\{\omega_{s} \mid s<n\right\}$.

It is clear that $A_{0}$ satisfies these conditions with $n=0$. 
Condition (3) will be used to obtain local finiteness, and condition (4) will assure that we have the descending chain condition for subalgebras. To see this suppose

$$
A_{n} \supset_{s} B_{0} \supset_{s} B_{1} \supset_{s} \cdots \text {. }
$$

Take $\xi_{i} \in B_{i}-B_{i+1}$. Then applying (4) to $\left\{\xi_{i} \mid i \in \omega\right\}$ we find that there is a $k_{0} \in \omega$ for which $\xi_{k_{0}} \in B_{k_{0}+1}$, a contradiction.

We now proceed to construct $A_{n+1}$ which satisfies conditions (1)(4) with $n$ replaced by $n+1$. For each $\xi$ with $\omega_{n} \leqq \xi<\omega_{n+1}$ we let $\phi_{\xi}$ map $\xi$ onto $\omega_{n}$ in a one-to-one manner with $\phi_{\omega_{n}}$ just the identity map on $\omega_{n}$. For each $m \in \omega$ we define $f_{m, n+1}$ as follows: If $\omega_{n} \leqq$ $\bigcap_{i=0}^{r(n)-1} \xi_{i}$; if $\eta_{i}<\xi_{i}$ for $i=0,1, \cdots, r(n)-1$; if $\omega_{n} \leqq \gamma$; and if

$$
\begin{aligned}
& \phi_{r}^{-1}\left(f_{m, n}\left(\phi_{\xi_{0}}\left(\eta_{0}\right), \cdots, \phi_{\xi_{r(n)-1}}\left(\eta_{r(n)-1}\right)\right)\right) \\
& \leqq\left(\left\{\eta_{0}, \cdots, \eta_{r(n)-1}, \xi_{0}, \cdots, \xi_{r(n)-1}\right\}\right. \\
&\left.-\left\{\omega_{s} \mid s \leqq n\right\}\right) ;
\end{aligned}
$$

we define

$$
\begin{aligned}
& f_{m, n+1}\left(\xi_{0}, \cdots, \xi_{r(n)-1}, \eta_{0}, \cdots, \eta_{r(n)-1}, \gamma\right) \\
& =\phi_{\gamma}^{-1}\left(f_{m, n}\left(\phi_{\xi_{0}}\left(\eta_{0}\right), \cdots, \phi_{\xi_{r(n)-1}}\left(\eta_{r(n)-1}\right)\right)\right) .
\end{aligned}
$$

Otherwise we define

$$
\begin{aligned}
& f_{m, n+1}\left(\xi_{0}, \cdots, \xi_{r(n)-1}, \eta_{0}, \cdots, \eta_{r(n)-1}, \gamma\right) \\
& =\cap\left\{\eta_{0}, \cdots, \eta_{r(n)-1}, \xi_{0}, \cdots, \xi_{r(n)-1}, \gamma\right\} .
\end{aligned}
$$

We let $A_{n+1}=\left\langle\omega_{n+1} ; f_{m, n+1}, \omega_{l}\right\rangle_{\substack{m \in \omega \\ l \leqq n}}$.

It is clear that $A_{n+1}$ satisfies conditions (1) and (3) of the induction hypothesis.

We now show that $A_{n+1}$ is locally finite. Suppose $B$ is a finite subset of $\omega_{n+1}$. Let

$$
\begin{aligned}
& B_{0}=B \cup\left\{\omega_{s} \mid s \leqq n\right\}, \\
& \vdots \\
& B_{k+1}=\left\{f_{m, n+1}\left(\xi_{0}, \cdots, \xi_{r(n+1)-1}\right) \mid m \in \omega \text { and } \xi_{0}, \cdots, \xi_{r(n+1)-1} \in \bigcup_{i \leqq k} B_{i}\right\} .
\end{aligned}
$$

Then $[B]=\bigcup_{k \in \omega} B_{k}$. In showing that $[B]$ is finite, we first show that each $B_{k}$ is finite. This is true for $k=0$. Assume that it is true for $k \leqq l$. Then $\bigcup_{i \leqq l} B_{i}$ is finite. Fix $\xi_{0}, \cdots, \xi_{r(n+1)-1} \in \bigcup_{i \leqq l} B_{i}$. Now we have

$$
\begin{aligned}
& \left\{f_{m, n+1}\left(\xi_{0}, \cdots, \xi_{r(n+1)-1}\right) \mid m \in \omega\right\} \\
& \subseteq \phi_{\xi_{r(n+1)-1}}^{-1}\left\{f_{m, n}\left(\phi_{\xi_{0}}\left(\xi_{r(n)}\right), \cdots, \phi_{\xi_{r(n)-1}}\left(\xi_{r(n+1)-1}\right)\right) \mid m \in \omega\right\} \\
& \quad \cup\left\{\xi_{0} \cap \cdots \cap \xi_{r(n+1-1)}\right\}
\end{aligned}
$$


However, this set is finite since $A_{n}$ is locally finite. Hence $B_{l+1}$ is finite, and by induction each $B_{k}$ is finite. Now let $C_{0}=B_{0}$ and

$$
C_{k+1}=B_{k+1}-B_{k} \text {. }
$$

Then $[B]=\mathrm{U}_{k \in \omega} C_{k}$, and each $C_{k+1}$ is finite. If $1 \leqq k<k^{\prime}$ and if $C_{k}, C_{k^{\prime}} \neq \varnothing$, then using (3) and the fact that $\left\{\omega_{s} \mid s \leqq n\right\} \subseteq B_{0}$, we see that $\max C_{k^{\prime}}<\max C_{k}$. Thus there are only finitely many $C_{k} \neq \varnothing$. Hence $[B]$ is finite.

Finally we show that $A_{n+1}$ satisfies condition (4). Suppose we have $\left\{\xi_{k} \mid k \in \omega\right\}$ a sequence of distinct elements of $\omega_{n+1}$. We consider two cases.

Case 1. There are infinitely many $k^{\prime} \mathrm{s}$ for which $\xi_{k} \in \omega_{n}$ : Without loss of generality we assume that $\left\{\xi_{k} \mid k \in \omega\right\} \subseteq \omega_{n}$. We then invoke the induction hypothesis to get an $m \in \omega$ and $k_{0}, k_{1}, \cdots, k_{r(n)} \in \omega$ so that $k_{0}<\bigcap_{i=1}^{r(n)} k_{i}$ and $f_{m, n}\left(\eta_{k_{1}}, \cdots, \eta_{k_{r(n)}}\right)=\xi_{k_{0}}$ where either $\eta_{k_{i}}=\xi_{k_{i}}$ or else $\eta_{k_{i}} \in\left\{\omega_{s} \mid s<n\right\}$. But then we have

$$
\begin{aligned}
& f_{m, n+1}\left(\omega_{n}, \cdots, \omega_{n}, \eta_{k_{1}}, \cdots, \eta_{k_{r(n)}}, \omega_{n}\right) \\
& \quad=\phi_{\omega_{n}}^{-1}\left(f_{m, n}\left(\phi_{\omega_{n}}\left(\eta_{k_{1}}\right), \cdots, \phi_{\omega_{n}}\left(\eta_{k_{r(n)}}\right)\right)\right) \\
& \quad=f_{m, n}\left(\eta_{k_{1}}, \cdots, \eta_{k_{r(n)}}\right) \\
& \quad=\xi_{k_{0}} .
\end{aligned}
$$

This completes the proof in this case.

Case 2. At most finitely many of the $\xi_{k}$ 's are less than $\omega_{n}$ : Without loss of generality we assume that $\left\{\xi_{k} \mid k \in \omega\right\} \cong \omega_{n+1}-\omega_{n}$. We pick $k_{0}<k_{1}<\cdots$ so that $\xi_{k_{0}}<\xi_{k_{1}}<\cdots$. For each $i \in \omega$, we let $\pi_{i}=\phi_{\xi_{k_{i+1}}}\left(\xi_{k_{i}}\right)$. Now consider $\left\{\pi_{i} \mid i \in \omega\right\}$. If for some $i, j \in \omega$ we have $i<j$ and $\pi_{i}=\pi_{j}$, then

$$
\begin{aligned}
f_{0, n+1} & \left(\xi_{k_{j+1}}, \cdots, \xi_{k_{j+1}}, \xi_{k_{j}}, \cdots, \xi_{k_{j}}, \xi_{k_{i+1}}\right) \\
& =\phi_{\xi_{k_{i+1}}}^{-1}\left(f_{0, n}\left(\phi_{\xi_{k_{j+1}}}\left(\xi_{k_{j}}\right), \cdots, \phi_{\xi_{k_{j+1}}}\left(\xi_{k_{j}}\right)\right)\right) \\
& =\phi_{\xi_{k_{i+1}}^{-1}}^{-1}\left(f_{0, n}\left(\pi_{j}, \cdots, \pi_{j}\right)\right) \\
& =\phi_{\xi_{k_{i+1}}}^{-1}\left(\pi_{j}\right) \\
& =\phi_{\xi_{k_{i+1}}}^{-1}\left(\pi_{i}\right) \\
& =\phi_{\xi_{k_{i+1}}}^{-1} \phi_{\xi_{k_{i+1}}}\left(\xi_{k_{i}}\right) \\
& =\xi_{k_{i}}
\end{aligned}
$$

and we're through. Thus we may assume that $\left\{\pi_{i} \mid i \in \omega\right\}$ is a sequence of distinct elements of $\omega_{n}$. Applying the induction hypothesis again, we get an $m \in \omega$ and $i_{0}, i_{1}, \cdots, i_{r(n)} \in \omega$ so that $i_{0}<\bigcap_{j=1}^{r(n)} i_{j}$ and

$$
f_{m, n}\left(\eta_{i_{1}}, \cdots, \eta_{i_{r(n)}}\right)=\pi_{i_{0}}
$$


where either $\eta_{i_{j}}=\pi_{i_{j}}$ or else $\eta_{i_{j}} \in\left\{\omega_{s} \mid s<n\right\}$. Now let

$$
\beta_{i_{j}}=\left\{\begin{array}{l}
\xi_{k_{i j}} \text { if } \eta_{i_{j}}=\pi_{i_{j}}, \\
\eta_{i_{j}} \text { otherwise }
\end{array}\right.
$$

and let

$$
\sigma_{i_{j}}=\left\{\begin{array}{l}
\xi_{k_{i_{j}+1}} \text { if } \beta_{i_{j}}=\xi_{k_{i_{j}}} \\
\omega_{n} \text { otherwise }
\end{array}\right.
$$

Then $\phi_{\sigma_{i_{j}}}\left(\beta_{i_{j}}\right)=\eta_{i_{j}}$ in any case. This gives

$$
\begin{aligned}
f_{m, n+1} & \left(\sigma_{i_{1}}, \cdots, \sigma_{i_{r(n)}}, \beta_{i_{1}}, \cdots, \beta_{i_{r(n)}}, \xi_{k_{i_{0}+1}}\right) \\
& =\phi_{\xi_{k_{0}+1}}^{-1}\left(f_{m, n}\left(\phi_{\sigma_{i_{1}}}\left(\beta_{i_{1}}\right), \cdots, \phi_{i_{i_{r(n)}}}\left(\beta_{\left.i_{r(n)}\right)}\right)\right)\right. \\
& =\phi_{\xi_{i_{0}+1}}^{-1}\left(f_{m, n}\left(\eta_{i_{1}}, \cdots, \eta_{i_{r(n)}}\right)\right) \\
& =\phi_{\xi_{k_{k_{0}+1}}}^{-1}\left(\pi_{i_{0}}\right) \\
& =\phi_{\xi_{k_{i_{0}+1}}}^{-1} \phi_{\xi_{k_{i_{0}+1}}}\left(\xi_{k_{i_{0}}}\right) \\
& =\xi_{k_{i_{0}}} .
\end{aligned}
$$

Since each $\sigma_{i_{j}}, \beta_{i_{j}}$ is a $\xi_{k_{i}}$ with $i>i_{0}$ or is in $\left\{\omega_{s} \mid s \leqq n\right\}$, this is the desired result. This completes the proof of Theorem 3.3.

COROLlaRY 3.4. For $n \in \omega$ there is a locally finite algebra of power $\omega_{n}$ which has one commutative binary operation and satisfies the descending chain condition for subalgebras.

\section{BIBLIOGRAPHY}

1. P. Erdos, and A. Hajnal, On a problem of B. Jónsson, Bulletin de L'Academie Polonaise de Sciences 14 (1966), 19-23.

2. W. Hanf, Representations of lattices by subalgebras (preliminary report), Bull. Amer. Math. Soc. 62 (1956), 402.

3. A. Kostinsky, Recent results on Jónsson algebras, seminar notes, Department of Mathematics, University of California at Berkeley, 1966.

4. T. Whaley, Algebras satisfying the descending chain condition for subalgebras, Vanderbilt University Dissertation, 1968.

5 . Infinite algebras satisfying the descending chain condition (preliminary report), Notices of the Amer. Math. Soc. 14 (1967), 714.

Received June 16, 1968. This work was supported in part by a National Science Foundation Grant GP-7252. These results are taken from the author's doctoral thesis, supervised by Professor B. Jónsson at Vanderbilt University. 



\section{PACIFIC JOURNAL OF MATHEMATICS}

\section{EDITORS}

H. ROYDEN

Stanford University

Stanford, California

R. R Phelps

University of Washington

Seattle, Washington 98105
J. DUGUNDJI

Department of Mathematics

University of Southern California

Los Angeles, California 90007

RICHARD ARENS

University of California

Los Angeles, California 90024

\section{ASSOCIATE EDITORS}
E. F. BECKENBACH
B. H. NeumanN
F. WOLF
K. YosidA

\section{SUPPORTING INSTITUTIONS}

UNIVERSITY OF BRITISH COLUMBIA

CALIFORNIA INSTITUTE OF TECHNOLOGY

UNIVERSITY OF CALIFORNIA

MONTANA STATE UNIVERSITY

UNIVERSITY OF NEVADA

NEW MEXICO STATE UNIVERSITY

OREGON STATE UNIVERSITY

UNIVERSITY OF OREGON

OSAKA UNIVERSITY

UNIVERSITY OF SOUTHERN CALIFORNIA
STANFORD UNIVERSITY

UNIVERSITY OF TOKYO

UNIVERSITY OF UTAH

WASHINGTON STATE UNIVERSITY

UNIVERSITY OF WASHINGTON

AMERICAN MATHEMATICAL SOCIETY CHEVRON RESEARCH CORPORATION TRW SYSTEMS

NAVAL WEAPONS CENTER 


\section{Pacific Journal of Mathematics

Vol. 28, No. $1 \quad$ March, 1969

Patrick Robert Ahern, On the geometry of the unit ball in the space of real annihilating measures .............................. 1

Kirby Alan Baker, Equational classes of modular lattices ............. 9

E. F. Beckenbach and Gerald Andrew Hutchison, Meromorphic minimal surfaces ......................................... 17

Tae Ho Choe, Intrinsic topologies in a topological lattice ..............

John Bligh Conway, A theorem on sequential convergence of measures and

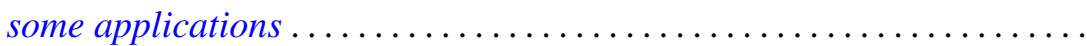

Roger Cuppens, On the decomposition of infinitely divisible probability laws without normal factor.

Lynn Harry Erbe, Nonoscillatory solutions of second order nonlinear

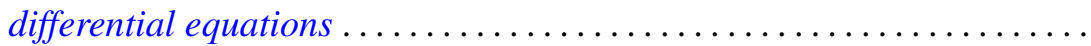

Burton I. Fein, The Schur index for projective representations of finite

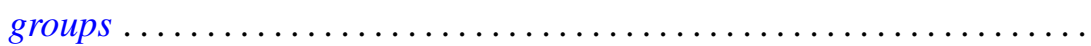

Stanley P. Gudder, A note on proposition observables............... 101

Kenneth Kapp, On Croisot's theory of decompositions ............... 105

Robert P. Kaufman, Gap series and an example to Malliavin's theorem . . . 117

E. J. McShane, Robert Breckenridge Warfield, Jr. and V. M. Warfield,

Invariant extensions of linear functionals, with applications to measures and stochastic processes ................

Marvin Victor Mielke, Rearrangement of spherical modifications ...

Akio Osada, On unicity of capacity functions ..............

Donald Steven Passman, Some 5/2 transitive permutation groups ...

Harold L. Peterson, Jr., Regular and irregular measures on groups and dyadic spaces...

Habib Salehi, On interpolation of $q$-variate stationary stochastic processes...

Michael Samuel Skaff, Vector valued Orlicz spaces generalized

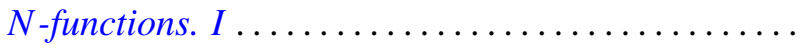

A. J. Ward, On $H$-equivalence of uniformities. II...........

Thomas Paul Whaley, Algebras satisfying the descending chain condition for subalgebras...

G. K. White, On subgroups of fixed index

Martin Michael Zuckerman, A unifying condition for implications among the axioms of choice for finite sets ................. 